\title{
MICROPARTÍCULAS NANORREVESTIDAS CONTENDO UM FÁRMACO MODELO HIDROFÓBICO: PREPARAÇÃO EM ETAPA ÚNICA E CARACTERIZAÇÃO BIOFARMACÊUTICA
}

\author{
Gislane Scholze Domingues e Sílvia Stanisçuaski Guterres* \\ Faculdade de Farmácia, Universidade Federal do Rio Grande do Sul, Av. Ipiranga, 2752, 90610-000 Porto Alegre - RS, Brasil \\ Ruy Carlos Ruver Beck \\ Departamento de Farmácia Industrial, Centro de Ciências da Saúde, Universidade Federal de Santa Maria, Av. Roraima, 1000, \\ 97105-900 Santa Maria - RS, Brasil \\ Adriana Raffin Pohlmann \\ Instituto de Química, Universidade Federal do Rio Grande do Sul, Av. Bento Gonçalves, 9500, 91501-970 Porto Alegre - RS, \\ Brasil
}

Recebido em 22/8/07; aceito em 12/6/08; publicado na web em 31/10/08

\begin{abstract}
NANOCAPSULE-COATED MICROPARTICLES PREPARED IN ONE STEP: CHARACTERIZATION AND IN VITRO DRUG RELEASE PROFILES EVALUATION. The aim of this work was to prepare and characterize spray-dried nanocapsule-coated microparticles obtained in one step, using indomethacin as a hydrophobic drug model and poly(ع-caprolactone) or Eudragit ${ }^{\circledR}$ RS100, as polymers. Nanocapsule-coated microparticles showed micrometric mean sizes $(10-15 \mu \mathrm{m})$ and a reduced surface area $(75-85$ $\left.\mathrm{m}^{2} \mathrm{~g}^{-1}\right)$ compared to the raw material $\left(214 \mathrm{~m}^{2} \mathrm{~g}^{-1}\right)$. Microparticles coated with Eudragit ${ }^{\circledast} \mathrm{RS} 100$-nanocapsules showed a better control of the drug release. The release profiles fit to the monoexponetial model and to the Power Law. The mechanism of the indomethacin release from the microparticles is non-Fickian and depends on the particles desagglomeration.
\end{abstract}

Keywords: indomethacin; microparticles; nanocapsules.

\section{INTRODUÇÃO}

No âmbito farmacêutico, os sistemas coloidais poliméricos caracterizam-se como partículas poliméricas de tamanho nanométrico (nanopartículas) contendo um fármaco, que se encontram suspensas em uma fase aquosa. Esses sistemas têm demonstrado muitas potencialidades no aumento da eficácia de fármacos, na diminuição da toxicidade ou, ainda, no desenvolvimento de sistemas de liberação modificada. ${ }^{1}$ Devido principalmente à sua fase aquosa, estas suspensões coloidais são bastante suscetíveis a alterações físico-químicas e contaminação microbiológica durante o armazenamento., ${ }^{2,3}$

Nesse sentido, a secagem por aspersão (spray-drying) foi proposta por nosso grupo de pesquisa para a conversão desses sistemas dispersos aquosos em sistemas sólidos microparticulados, empregando o dióxido de silício coloidal (Aerosil ${ }^{\circledR} 200$ ) como adjuvante de secagem. Considerando que os fármacos antiinflamatórios não-esteróides têm sido foco de nossas pesquisas e que sua nanoencapsulação permite a redução significativa dos efeitos tóxicos de fármacos desta classe sobre a mucosa gastrintestinal, ${ }^{4-6}$ demonstramos a aplicação desta técnica de secagem para a obtenção de produtos secos microparticulados a partir de diferentes tipos de nanopartículas poliméricas contendo indometacina ou diclofenaco. ${ }^{6,7}$ Estes produtos secos são compostos pelo dióxido de silício coloidal, na forma de aglomerados micrométricos, revestidos com nanopartículas poliméricas (nanocápsulas - estrutura vesicular ou nanoesferas - estrutura matricial). ${ }^{7,8}$

De acordo com esses trabalhos, ${ }^{4-8}$ as suspensões de nanopartículas (nanocápsulas ou nanoesferas), contendo um fármaco associado, empregadas nos estudos de secagem apresentavam diâmetros médios entre 200 e $300 \mathrm{~nm}$, quando avaliadas por espalhamento de luz dinâmico na forma líquida original. No caso da secagem de suspensões de nanocápsulas, as análises morfológicas por microscopia eletrônica

\footnotetext{
*e-mail: nanoc@farmacia.ufrgs.br
}

de varredura mostraram após aspersão a presença de nanoestruturas na superfície das micropartículas com tamanhos compatíveis àqueles determinados nas suspensões originais..$^{6-9} \mathrm{O}$ modelo esquemático para esses sistemas encontra-se representado na Figura 1a. Por outro lado, Pohlmann e colaboradores, ${ }^{8}$ após a secagem de suspensão de nanoesferas, observaram uma diminuição no diâmetro das nanoestruturas presentes na superfície das micropartículas, em relação ao diâmetro determinado na forma líquida original, evidenciando a ocorrência da alteração da sua organização estrutural durante a aspersão. Esta alteração foi atribuída à liberação do tensoativo lipofílico durante o processo de secagem, que se encontrava disperso na matriz polimérica das nanoesferas, com conseqüente redução no diâmetro das nanoestruturas adsorvidas à superfície dos pós. Esta alteração estrutural decorrente da secagem de suspensões de nanoesferas resultou em um impacto importante sobre a atividade biológica dos sistemas, como demonstrado através de estudos comparativos da tolerância gastrintestinal em ratos, de nanocápsulas e nanoesferas contendo indometacina ou diclofenaco antes e depois da secagem; ${ }^{4,5,10}$ enquanto que tanto as nanocápsulas quanto as nanoesferas foram eficientes na diminuição da toxicidade gastrintestinal dos fármacos quando em suspensão aquosa, somente os produtos secos contendo nanocápsulas mantiveram esta propriedade protetora frente aos efeitos ulcerativos dos fármacos.

Após os relatos do nosso grupo a respeito do desenvolvimento, caracterização e aplicação da técnica de secagem por aspersão para a obtenção de produtos secos contendo nanopartículas poliméricas, outros grupos de pesquisa têm se dedicado ao estudo destes sistemas, adotando a secagem por aspersão como alternativa para transpor a limitada estabilidade físico-química das suspensões de nanopartículas $^{11,12}$ ou, ainda, desenvolver sistemas microparticulados de administração intrapulmonar de fármacos. ${ }^{13}$

Com relação às potencialidades no âmbito tecnológico, Müller ${ }^{14}$ demonstrou o emprego destes produtos secos obtidos a partir da 
secagem de suspensões de nanocápsulas por aspersão, como intermediários para a preparação de formas farmacêuticas derivadas, como cápsulas de gelatina dura. Entretanto, torna-se importante ressaltar que esta estratégia é aplicável a fármacos de elevada potência, normalmente administrados em baixas dosagens. Isto é explicado pela limitada capacidade de carga das nanocápsulas, o que leva à obtenção de produtos secos (micropartículas de nanocápsulas e dióxido de silício aglomeradas) com baixa dosagem de substância ativa, não atingindo a dose terapêutica.

Visando contornar essa limitação, uma segunda estratégia foi concebida (Figura 1b), associando o fármaco ao adjuvante de secagem e empregando a suspensão nanoestruturada apenas como material de revestimento e sistema controlador da liberação do fármaco. ${ }^{15}$ Esses novos sistemas foram chamados de micropartículas revestidas com nanopartículas (nanoparticle-coated microparticles). Até o momento, esta segunda estratégia foi estudada e aplicada a dois modelos de fármacos, o diclofenaco e a dexametasona, cujas micropartículas foram revestidas com suspensões de nanocápsulas, nanoesferas ou nanodispersão, empregando Eudragit ${ }^{\circledR}$ S100 como polímero. ${ }^{15-18}$ No desenvolvimento desta nova estratégia foram relatados dois métodos de preparação para as micropartículas nanorrevestidas. $\mathrm{O}$ primeiro chamado de método de preparação em etapa única foi aplicado ao modelo hidrofílico (diclofenaco sódico), enquanto que o segundo chamado de método em duas etapas foi aplicado aos modelos hidrofílico e hidrofóbico (diclofenaco sódico e diclofenaco ácido). ${ }^{15}$ a)

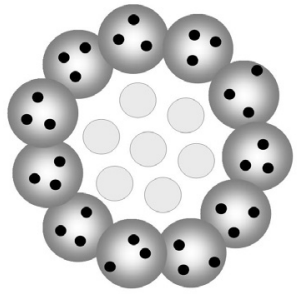

- Fármaco

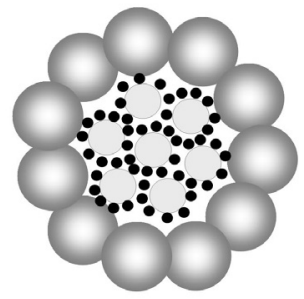

Nanopartículas

Figura 1. Representação esquemática das micropartículas nanorrevestidas, fármaco associado às nanopartículas (a) ou ao adjuvante de secagem (b)

De acordo com os resultados demonstrados por Beck e colaboradores,${ }^{15} \mathrm{o}$ maior potencial, considerando-se o controle da liberação do fármaco pela camada de nanopartículas revestindo o núcleo contendo o fármaco, foi demonstrado para as formulações preparadas em duas etapas, tanto para o modelo hidrofílico quanto para o hidrofóbico. Em relação ao sistema de revestimento, os melhores resultados no controle da liberação e da permeação do fármaco através de células Caco- 2 e na capacidade protetora, frente ao efeito irritativo do diclofenaco sobre a mucosa gastrintestinal, foram demonstrados pelas micropartículas revestidas a partir de suspensões de nanocápsulas. ${ }^{15,17,18}$

Considerando que até o momento a estratégia do revestimento de micropartículas a partir de suspensões de nanopartículas, empregadas como material de revestimento, através do método de preparação em etapa única, foi aplicada somente a um modelo de fármaco hidrofílico, os objetivos deste trabalho foram preparar e caracterizar micropartículas nanorrevestidas em etapa única, empregando um fármaco modelo hidrofóbico, a indometacina (Figura 2). Suspensões de nanocápsulas foram empregadas como material de revestimento devido a suas maiores potencialidades demonstradas nos estudos anteriores. ${ }^{5,15}$ Além disso, foi avaliada a influência do emprego de dois polímeros diferentes na preparação destas nanocápsulas sobre as características físico-químicas das micropartículas nanorrevestidas: a poli(ع-caprolactona), um poliéster biodegradável e o Eudragit ${ }^{\circledR} \mathrm{RS}$ 100, um copolímero acrílico (Figura 2). As micropartículas nanorrevestidas foram caracterizadas quanto ao teor de fármaco, diâmetro médio e potencial zeta após ressuspensão, análise morfológica (MEV), determinação da área superficial e tamanho de poros, análise granulométrica e avaliação do perfil de liberação in vitro do fármaco. Os perfis de liberação foram analisados matematicamente através de métodos modelo-dependentes, para se determinar a velocidade de liberação da indometacina e aplicando-se, pela primeira vez para esses sistemas, a Lei da Potência (modelo de Korsmeyer-Peppas) para descrever o mecanismo de liberação do fármaco a partir de micropartículas nanorrevestidas. a)<smiles>COc1ccc2c(c1)c(CC(=O)O)c(C)n2C(=O)c1ccc(Cl)cc1</smiles>

b)

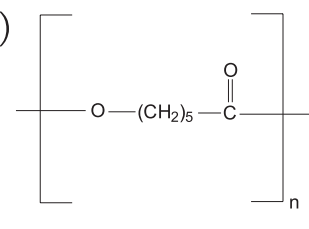

c)

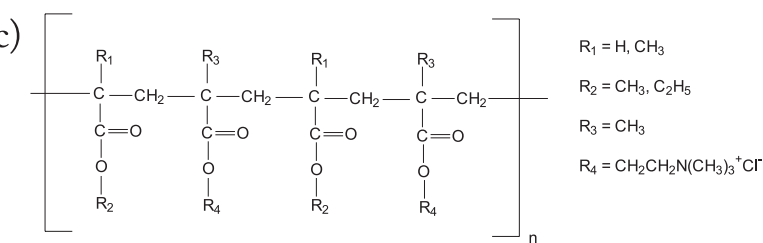

Figura 2. Estrutura molecular da (a) indometacina, (b) poli(E-caprolactona) e (c) Eudragit ${ }^{\boxplus}$ RS 100

\section{PARTE EXPERIMENTAL}

\section{Materiais}

Indometacina foi obtida da Sigma (St. Louis, EUA); os polímeros, poli(E-caprolactona) (PCL, MM $65000 \mathrm{Da})$ da Aldrich (Strasbourg, França) e poli(acrilato de etila-co-metacrilato de metila-co-metacrilato de trimetilamônio etila clorídrico) (Eudragit RS ${ }^{\circledast} 100$, ERS, MM 150000 Da) da Degussa (São Paulo, Brasil). Os tensoativos monooleato de sorbitano e polissorbato 80 foram adquiridos da Delaware (Porto Alegre, Brasil) e a mistura de triglicerídeos dos ácidos cáprico e caprílico da Brasquim (Porto Alegre, Brasil). O dióxido de silício coloidal (Aerosil ${ }^{\circledR}$ 200) foi adquirido da Degussa (São Paulo, Brasil). Os demais reagentes e solventes empregados apresentaram grau analítico ou cromatográfico, dependendo da finalidade e foram utilizados como recebidos.

\section{Métodos}

\section{Preparação das suspensões de nanocápsulas}

As suspensões de nanocápsulas foram preparadas $(n=3)$ utilizando o método da deposição interfacial dos polímeros pré-formados, descrito por Fessi e colaboradores..$^{19}$ A fase orgânica composta de acetona (267 mL), polímero (1000 mg, PCL ou ERS), monooleato de sorbitano (383 mg) e óleo (3102 mg) foi vertida na fase aquosa (533 mL) contendo polissorbato 80 (383 mg), sob agitação magnética moderada. $\mathrm{O}$ volume final foi reduzido a $100 \mathrm{~mL}$ de suspensão em um evaporador rotatório, a $40{ }^{\circ} \mathrm{C}$.

\section{Caracterização das suspensões de nanocápsulas}

O diâmetro médio das partículas em suspensão foi determinado (n $=3$ ) através de espalhamento de luz dinâmico (Zetasizer ${ }^{\circledR}$ Nanoseries, 
Malvern Instrumens, Inglaterra), diluindo-se as amostras 500 vezes em água purificada (sistema Milli- ${ }^{\circledR}$ ).

O potencial zeta foi determinado após diluição de 500 vezes das suspensões em solução de $\mathrm{NaCl} 1 \mathrm{mmol} \mathrm{L}^{-1}$ (Zetasizer $^{\circledR}$ Nanoseries, Malvern Instruments, Inglaterra). Foram utilizadas células capilares fechadas (DTS1060) para as leituras e as medidas foram realizadas em triplicata.

Os valores de $\mathrm{pH}$ das suspensões de nanocápsulas foram determinados diretamente nas suspensões através de um potenciômetro (Modelo B474, Micronal, Brasil), previamente calibrado com soluções-tampão pH 4,0 e 7,0.

Preparação em etapa única das micropartículas nanorrevestidas contendo indometacina

A indometacina ( $0,075 \mathrm{~g})$ e o dióxido de silício coloidal (1,5g) foram pesados e adicionados à suspensão $(50 \mathrm{~mL})$ de nanocápsulas vazias, mantendo-se sob agitação durante 3 min para posterior aspersão (Mini Spray Dryer MSD ${ }^{\circledR}$ 1.0, Labmaq, São Paulo, Brasil, segundo as condições operacionais descritas na Tabela 1. Foram preparadas formulações de micropartículas a partir de suspensões de nanocápsulas de PCL (M1) ou Eudragit ${ }^{\circledR}$ RS 100 (M2).

Tabela 1. Condições operacionais utilizadas na secagem por aspersão das micropartículas nanorrevestidas em equipamento $\mathrm{MSD}^{\circledR} 1.0$

\begin{tabular}{lc}
\hline Parâmetros & Condição \\
\hline Fluxo de alimentação & $0,2 \mathrm{~L} / \mathrm{h}$ \\
Fluxo de ar & $500 \mathrm{NL} / \mathrm{h}$ \\
Pressão de ar comprimido & $3 \mathrm{kgf} / \mathrm{cm}^{2}$ \\
Temperatura de entrada do ar de secagem & $150 \pm 5^{\circ} \mathrm{C}$ \\
Temperatura de saída do ar de secagem & $80 \pm 5^{\circ} \mathrm{C}$ \\
Capa de fluido (diâmetro & $0,8 \mathrm{~mm}$ \\
do bico atomizador) & $0,6 \mathrm{NMC}\left(0,6 \mathrm{~m}^{3} / \mathrm{min}\right)$ \\
\hline Sistema de aspiração
\end{tabular}

\section{Caracterização das micropartículas nanorrevestidas}

\section{Determinação do teor de umidade}

As micropartículas (M1 e M2) foram submetidas à avaliação por método coulométrico por Karl Fischer com reagente de Karl Fischer segundo a Farmacopéia Brasileira $4^{\mathrm{a}}$ ed. ${ }^{20}$

\section{Determinação do teor de indometacina}

A quantificação da indometacina nos pós foi realizada através de cromatografia líquida de alta eficiência (CLAE). A detecção foi realizada na região do UV a $254 \mathrm{~nm}$, sendo a fase móvel composta por acetonitrila/água $(65: 35 \mathrm{v} / \mathrm{v})$, contendo $50 \mathrm{mmol} \mathrm{L}^{-1}$ trietilamina, cujo $\mathrm{pH}$ foi ajustado a $\mathrm{pH}$ 3,3 (aparente) com ácido fosfórico. O fluxo da fase móvel foi de $1,0 \mathrm{~mL} \mathrm{~min}^{-1}$ e o volume de injeção de $20 \mu \mathrm{L} .{ }^{21}$ Foi utilizado um cromatógrafo Perkin Elmer S-200 com detector UV-VIS e injetor S-200. A fase estacionária empregada foi uma coluna Nova-Pak C18 Waters (3,9 X $150 \mathrm{~mm})$. O método analítico foi validado de acordo com os seguintes parâmetros: ${ }^{22}$ linearidade ( $\mathrm{y}=92460 \mathrm{x}-36109, r^{2}=0,999, \mathrm{n}=6$ ), faixa de concentração entre $1 \mathrm{e} 40 \mu \mathrm{g} \mathrm{mL}^{-1}$, precisão (DPR intra-dia $=1,55-1,95 \%$; DPR interdia $=1,68 \%$ ), exatidão $(99,93 \pm 1,02 \%)$ e especificidade (não houve interferência dos adjuvantes na quantificação da indometacina).

A preparação da amostra foi realizada pela adição da acetonitrila sobre uma quantidade determinada de pó, seguida por uma agitação magnética durante 120 min e centrifugação a $3500 \mathrm{~g}$ durante $10 \mathrm{~min}$. A solução sobrenadante foi filtrada a $0,45 \mu$ m e injetada no sistema cromatográfico.
Determinação do diâmetro após ressuspensão em água

O diâmetro médio das partículas foi determinado após dispersão do pó em água (em volume equivalente ao das suspensões originais). $\mathrm{O}$ diâmetro foi determinado em Zetasizer ${ }^{\circledR}$ (Nanoseries, Malvern, Inglaterra). ${ }^{9}$ As micropartículas nanorrevestidas redispersas em água foram filtradas através de membrana $0,45 \mu \mathrm{m}$ e diluídas com água purificada (sistema Milli-Q ${ }^{\circledR}$ ). As medidas foram realizadas em dispositivo de poliestireno $(n=3)$.

\section{Determinação do potencial zeta após ressuspensão em água}

O potencial zeta foi determinado $(n=3)$ após dispersão dos pós em água (Zetasizer ${ }^{\circledR}$ Nanoseries, Malvern Instruments, Inglaterra), empregando volume equivalente ao das suspensões originais. ${ }^{9}$ As dispersões aquosas de micropartículas nanorrevestidas foram filtradas $(0,45 \mu \mathrm{m})$ e diluídas em solução de $\mathrm{NaCl} 1 \mathrm{mmol} \mathrm{L}^{-1}$.

\section{Avaliação morfológica através de microscopia eletrônica de varredura}

A análise microscópica (superfície e forma) dos pós foi realizada através de microscopia eletrônica de varredura (Jeol Scanning Microscope, JSM-5800, Toquio, Japão). As amostras foram previamente metalizadas com ouro (Jeol Jee 4BSVG-IN, Tóquio, Japão) e as fotomicrografias foram obtidas utilizando-se uma voltagem de $20 \mathrm{kV}$ e aumentos entre 1000 a 45000 vezes. As análises foram realizadas no Centro de Microscopia Eletrônica da UFRGS.

\section{Determinação da área superficial e da distribuição de tamanho de poros}

As áreas superficiais e a distribuição do tamanho de poros das micropartículas e do Aerosil ${ }^{\circledR} 200$ foram determinadas nas amostras previamente desgaseificadas, em equipamento volumétrico, utilizando nitrogênio como gás de adsorção-dessorção. Foi empregado um equipamento de fabricação própria (Laboratório de Sólidos e Superfícies, Instituto de Química, UFRGS) dotado de um sistema de vácuo, obtido pelo emprego de uma bomba de vácuo turbomolecular (Edwards, West Sussex, Inglaterra). As medidas de pressão foram determinadas utilizando-se um barômetro capilar de mercúrio e os resultados comparados a um padrão de referência de alumina. A análise dos resultados foi realizada utilizando o método BET (Brunauer, Emmett e Teller) ${ }^{23}$ e o método BJH (Barret, Joyner, Halenda), ${ }^{24}$ para a determinação da área superficial e da distribuição de poros, respectivamente.

\section{Análise granulométrica}

A análise granulométrica das amostras foi realizada a seco por difratometria a laser através de um equipamento Beckman Coulter ${ }^{\circledR}$ Tornado (Beckmann Instruments, USA). A análise foi realizada no Laboratório de Tecnologia de Partículas do Instituto de Pesquisas Tecnológicas, IPT/SP. O valor de SPAN foi empregado como medida da dispersão granulométrica, o qual relaciona os valores encontrados de diâmetros das partículas correspondentes a 10, 50 e 90\% da distribuição acumulada para uma amostra. As medidas de $\mathrm{d}_{(4,3)}$ foram calculadas a partir das determinações dos volumes médios das partículas. ${ }^{25}$

\section{Avaliação do perfil de liberação in vitro da indometacina}

$\mathrm{O}$ perfil de liberação in vitro do fármaco foi avaliado $(\mathrm{n}=3)$ através de um equipamento dotado de célula de fluxo. Este equipamento consistiu em células de vidro contendo uma membrana de fibra de vidro (AP25, Millipore), conectadas a uma bomba peristáltica (Miliplus 3, Gilson) através de cânulas de silicone. O meio de dissolução (intestinal simulado sem enzimas pH 6,8 ${ }^{26}$ ) foi mantido em banho termostatizado a $37,0 \pm 0,5^{\circ} \mathrm{C}$. O fluxo do meio de dissolução 
foi de $1 \mathrm{~mL} \mathrm{~min}{ }^{-1}$. Esta metodologia garante as condições sink ao experimento, ${ }^{27}$ por ser um sistema aberto de passagem do meio de liberação a um fluxo constante. A massa de micropartículas utilizada foi correspondente a $1,5 \mathrm{mg}$ de indometacina As amostras foram coletadas em tempos pré-determinados durante 200 min e analisadas através de CLAE, empregando a metodologia descrita anteriormente e validada para o teste de liberação in vitro, que demonstrou ser linear na faixa de concentração entre 1 e $40 \mu \mathrm{g} \mathrm{mL}^{-1}\left(\mathrm{y}=92460 \mathrm{x}-36109, r^{2}\right.$ $=0,999, \mathrm{n}=6$ ); precisa (DPR intra-dia e inter-dia menor que $2,0 \%$ ), exata $(101,52 \pm 2,63 \%)$ e específica (não houve interferência dos adjuvantes na quantificação da indometacina). ${ }^{22}$

Para a avaliação matemática dos perfis de liberação foram aplicados os métodos modelo-dependentes mono e biexponencial. ${ }^{28} \mathrm{~A}$ Lei da Potência foi aplicada para determinar o mecanismo de liberação do fármaco a partir das micropartículas. Este modelo semi-empírico relaciona exponencialmente a liberação de uma substância com o tempo, ${ }^{29}$ sendo o valor de $n$ utilizado para caracterizar o mecanismo de liberação. A adequabilidade dos modelos aos dados experimentais foi avaliada com o auxílio do programa MicroMath Scientist ${ }^{\circledR} 3.0$ (Micromath, EUA), comparando-se os modelos entre si com base nos seguintes parâmetros: critério de seleção de modelo (MSC), coeficiente de correlação $(r)$ e ajuste gráfico.

\section{RESULTADOS E DISCUSSÃO}

\section{Suspensões de nanocápsulas}

As suspensões de nanocápsulas apresentaram diâmetros médios compatíveis com os sistemas nanoestruturados preparados pelo método da deposição interfacial de polímeros pré-formados (Tabela 2). ${ }^{1,4}$ Conforme pode ser observado, as nanocápsulas preparadas com Eudragit $^{\circledR}$ RS 100 apresentaram diâmetro médio e valores de $\mathrm{pH}$ inferiores àqueles encontrados para as nanocápsulas preparadas com poli( $\varepsilon$-caprolactona). Estas diferenças estão de acordo com aquelas observadas anteriormente por Schaffazick e colaboradores ${ }^{30}$ no desenvolvimento de nanocápsulas contendo melatonina, empregando os mesmos polímeros.

Em relação ao potencial zeta (Tabela 2), as suspensões preparadas com Eudragit ${ }^{\circledR}$ RS 100 apresentaram valores positivos $(+23,4 \pm 1,5$ $\mathrm{mV}$ ), que podem ser atribuídos à carga positiva dos grupamentos amônio quaternário presentes no polímero. Este resultado corrobora aquele encontrado por Ubrich e colaboradores, ${ }^{31}$ que ao avaliarem o potencial zeta de nanocápsulas de Eudragit ${ }^{\circledR}$ RS e RL contendo ciclosporina, preparadas pelo mesmo método empregado em nosso estudo, também verificaram valores positivos do potencial zeta para estas formulações (entre +49 e $+63 \mathrm{mV}$ ). Por outro lado, as formulações preparadas com a poli( $\varepsilon$-caprolactona) apresentaram potencial zeta negativo $(-23,3 \pm 0,3 \mathrm{mV})$ decorrente da presença dos grupamentos éster do polímero e de acordo com valores anteriormente descritos na literatura para formulações preparadas com este polímero. ${ }^{9}$

\section{Micropartículas nanorrevestidas em etapa única}

As micropartículas nanorrevestidas contendo indometacina (M1 e M2) preparadas a partir das suspensões de nanocápsulas, previamente

Tabela 2. Características físico-químicas das suspensões de nanocápsulas (DP: desvio padrão referente à triplicata de lote)

\begin{tabular}{lccc}
\hline Polímero & $\begin{array}{c}\text { Diâmetro } \\
(\mathrm{nm} \pm \mathrm{DP})\end{array}$ & $\mathrm{pH} \pm \mathrm{DP}$ & $\begin{array}{c}\text { Potencial zeta } \\
(\mathrm{mV} \pm \mathrm{DP})\end{array}$ \\
\hline PCL & $374 \pm 12$ & $5,5 \pm 0,2$ & $-23,3 \pm 0,3$ \\
ERS & $161 \pm 3$ & $4,4 \pm 0,3$ & $+23,4 \pm 1,5$ \\
\hline
\end{tabular}

caracterizadas, apresentaram aspecto pulverulento, com valores de rendimento do processo entre 77 e $70 \%$ para as formulações M1 e M2, respectivamente. Valores semelhantes (70\%) foram relatados por Beck e colaboradores ${ }^{15}$ na preparação de micropartículas nanorrevestidas em etapa única, empregando um fármaco-modelo hidrofílico. Por outro lado, esses valores são superiores àqueles relatados para a obtenção destas micropartículas em duas etapas, contendo diclofenaco como fármaco hidrofóbico $\left(41-51 \%{ }^{15} ; 63 \%\right)^{17}$. As formulações apresentaram teores de umidade inferiores a $1 \%$, confirmando a eficiência do processo de secagem, e teores da indometacina entre 94,4 e $135,5 \%$, para as formulações M1 e M2, respectivamente. Os valores de recuperação acima de $100 \%$ para as formulações preparadas com o Eudragit ${ }^{\circledR}$ RS (M2), podem ser explicados pela segregação do pó durante a etapa de secagem, devido à adesão de componentes à câmara de secagem e conseqüente concentração do fármaco nos produtos finais, que está relacionada com as condições experimentais, ao tipo de sistema nanoestruturado e às características do fármaco. ${ }^{15,18}$

A observação morfológica das micropartículas nanorrevestidas através de MEV (Figura 3) em baixa magnificação demonstrou partículas esféricas e com ampla distribuição de tamanho para ambas as formulações (Figura 3, M1a e M2a). Através desta visualização pode ser observado um pequeno aumento no tamanho das micropartículas preparadas com Eudragit ${ }^{\circledR}$ RS 100 em relação àquelas preparadas com poli( $\varepsilon$-caprolactona). Entretanto, ambas as formulações demonstraram micropartículas com diâmetros menores que aqueles observados para o suporte de secagem, o dióxido de silício coloidal - Aerosil ${ }^{\circledR} 200$ comercial (Figura 3, Aa). A observação morfológica destas formulações em magnificações superiores (45.000x) permitiu a visualização de estruturas de diâmetro nanométrico revestindo a superfície das micropartículas compostas pelo Aerosil ${ }^{\circledR} 200$ e indometacina (Figura 3, M1b e M2b), enquanto que o suporte de secagem apresentou apenas uma superfície rugosa (Figura 3, Ab), corroborando os resultados previamente publicados empregando outros fármacosmodelo e diferentes métodos de preparação. ${ }^{15-18}$

Para se avaliar a influência deste nanorrevestimento sobre a área superficial, foram determinados o volume, o tamanho e a distribuição

M1
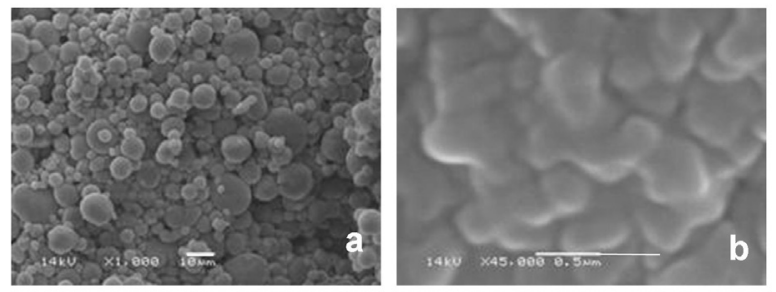

M2
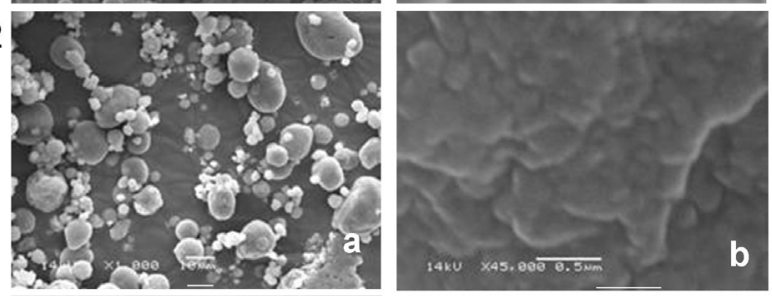

A
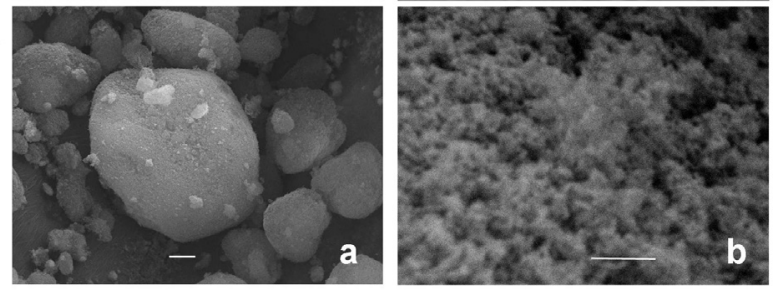

Figura 3. Fotomicrografias das formulações de micropartículas nanorrevestidas M1(PCL) e M2 (ERS); e do Aerosil ${ }^{\circledR} 200$ (A) [(a) largura da barra 10 $\mu \mathrm{m}$, (b) largura da barra $0,5 \mu \mathrm{m}]$ 
de poros das formulações empregando métodos baseados na isotermas de adsorção e dessorção de nitrogênio. Os resultados foram analisados empregando-se os métodos de BET e BJH. ${ }^{23,24}$ As áreas superficiais (Tabela 3) das formulações M1 e M2 foram semelhantes entre si, mas inferiores àquela apresentada pelo suporte de secagem (Aerosil ${ }^{\circledR}$ 200). Esta redução das medidas de área superficial pode ser atribuída à presença das nanoestruturas recobrindo os espaços interparticulares do Aerosil ${ }^{\circledR} 200 .{ }^{16-18}$ Resultados semelhantes foram encontrados por Beck e colaboradore ${ }^{17}$ para micropartículas contendo um fármaco hidrofóbico (diclofenaco) revestidas com nanocápsulas, através de um processo baseado em duas etapas $\left(61 \mathrm{~cm}^{2} \mathrm{~g}^{-1}\right)$. Entretanto, esta redução na área superficial também poderia estar relacionada a um aumento no tamanho de partículas das formulações. Através da análise conjunta dos valores de tamanho de partículas e área superficial (Tabela 3) é possível rejeitar esta última hipótese. As amostras M1 e M2 apresentaram tamanhos médios de aglomerados menores (10 e $15 \mu \mathrm{m}$, respectivamente) que os aglomerados de Aerosil $^{\circledR} 200$ (22 $\mu \mathrm{m})$ e as áreas superficiais de M1 e de M2 foram menores que a do Aerosi $1^{\circledR} 200$. Esses resultados demonstram que a diminuição da área foi devida ao nanorrevestimento das micropartículas. Além disso, ambas as formulações apresentaram volumes de poros inferiores $\left(0,093 \mathrm{~cm}^{3} \mathrm{~g}^{-1}\right.$ para M1 e $0,102 \mathrm{~cm}^{3} \mathrm{~g}^{-1}$ para M2) ao apresentado pelo Aerosil $^{\circledR} 200\left(0,307 \mathrm{~cm}^{3} \mathrm{~g}^{-1}\right)$, com distribuição de tamanho inferior a 6 $\mathrm{nm}$ (gráficos não apresentados), confirmando que o nanorrevestimento foi responsável pela diminuição da área superficial. Essa redução dos volumes de poros foi semelhante àquela relatada anteriormente para micropartículas nanorrevestidas a partir de nanocápsulas poliméricas, empregado um método de preparação em duas etapas $\left(0,04 \mathrm{~cm}^{3} \mathrm{~g}^{-1}\right)$ e um fármaco modelo hidrofóbico (diclofenaco). ${ }^{17}$

A análise granulométrica das formulações (Tabela 3) foi efetuada em equipamento Beckman Coulter ${ }^{\circledR}$ (Tornado) que determina os diâmetros dos pós a seco. O SPAN, que representa uma medida da dispersão granulométrica, foi de 2,45 para a formulação M1, de 1,76 para a formulação M2 e de 1,52 para o Aerosil ${ }^{\circledR} 200$. Valores de SPAN próximos ou inferiores a 2 indicam que as partículas podem ser consideradas adequadas para administração oral. ${ }^{23}$

Para se avaliar o comportamento dos sistemas após a ressuspensão em água, foram medidos os diâmetros médios e os potenciais zeta de dispersões aquosas dos pós microparticulados (Tabela 4). Analisandose os valores de diâmetros médios obtidos a partir dos filtrados das dispersões de micropartículas em água, pode-se observar que estes sistemas apresentam um comportamento de desaglomeração após

Tabela 3. Área superficial, volume de poros, diâmetro médio e distribuição granulométrica das micropartículas nanorrevestidas (formulações M1 - (PCL) e M2- (ERS) e do suporte de secagem (Aerosil ${ }^{\circledR}$ 200)

\begin{tabular}{lcccc}
\hline Formulação & $\begin{array}{c}\text { Área } \\
\text { superficial } \\
\left(\mathrm{m}^{2} \mathrm{~g}^{-1}\right)\end{array}$ & $\begin{array}{c}\text { Volume } \\
\text { de poros } \\
\left(\mathrm{cm}^{3} \mathrm{~g}^{-1}\right)\end{array}$ & $\mathrm{d}_{(4,3)} \mu \mathrm{m}^{*}$ & SPAN** $^{*}$ \\
\hline M1 & 78 & 0,093 & 10 & 2,4 \\
M2 & 85 & 0,102 & 15 & 1,8 \\
Aerosil $^{\circledR} 200$ & 214 & 0,307 & 22 & 1,5 \\
\hline
\end{tabular}

$* \mathrm{~d}_{(4,3)} \mu \mathrm{m}$ : diâmetro médio em volume; ${ }^{*}$ SPAN: dispersão granulométrica

Tabela 4. Diâmetros das partículas e potenciais zeta das formulações M1 (PCL) e M2 (ERS) após ressuspensão em água

\begin{tabular}{lcc}
\hline Formulação & Diâmetro $(\mathrm{nm} \pm \mathrm{DP})$ & Potencial zeta $(\mathrm{mV} \pm \mathrm{DP})$ \\
\hline M1 & $432 \pm 159$ & $-22,9 \pm 2,3$ \\
M2 & $368 \pm 332$ & $-11,5 \pm 4,2$ \\
\hline
\end{tabular}

a ressuspensão, dando origem a partículas com diâmetros médios entre 300 e $400 \mathrm{~nm}$, com ampla faixa de distribuição granulométrica, observada pela análise dos desvios-padrão determinados para ambas as amostras.

Em relação ao potencial zeta, enquanto que os valores determinados no filtrado da dispersão dos pós não apresentaram uma alteração significativa para as formulações preparadas com poli( $\varepsilon$-caprolactona) (M1) em relação às suspensões originais, a formulação preparada com Eudragit ${ }^{\circledR}$ RS 100 (M2) apresentou uma alteração significativa. $\mathrm{Na}$ suspensão original o valor de potencial zeta foi positivo $(+23,4 \pm$ $1,5 \mathrm{mV}$ ) enquanto que após a ressuspensão o respectivo filtrado apresentou um potencial zeta negativo $(-11,5 \pm 4,2 \mathrm{mV})$. Esta alteração pode ser atribuída à formação de sal do grupamento carboxílico da indometacina com os grupamentos amônio quaternários do polímero. A neutralização de cargas e o excesso de indometacina na superfície das estruturas poderiam levar à diminuição do potencial zeta. Para comprovar esta hipótese foi avaliado o potencial zeta das suspensões de nanocápsulas preparadas a partir do Eudragit ${ }^{\circledR}$ RS 100 contendo indometacina $(1,5 \mathrm{mg} / \mathrm{mL})$, que apresentaram um valor de $+19,2 \pm$ $2,4 \mathrm{mV}$. Através da comparação deste valor com aquele determinado para a suspensão de nanocápsulas para o revestimento, sem indometacina $(+23,4 \pm 1,5 \mathrm{mV})$, verificou-se uma tendência à diminuição do potencial zeta pela presença da indometacina. Além disso, o Aerosil ${ }^{\circledR}$ 200 presente nos pós também pode alterar o potencial de superfície das partículas. Para que essas afirmativas fossem verificadas, foram preparadas micropartículas nanorrevestidas a partir de nanocápsulas de Eudragit ${ }^{\circledR}$ RS 100, omitindo-se a adição da indometacina. Estas micropartículas foram ressuspensas em água, filtradas e analisadas, apresentando um potencial zeta de $-17,8 \pm 1,5 \mathrm{mV}$. Estes resultados demonstram que apesar da indometacina ocasionar uma diminuição do potencial zeta, a presença do Aerosil ${ }^{\circledR} 200$ é o fator mais importante na alteração (diminuição) dos valores de potencial zeta após a ressuspensão em água das micropartículas nanorrevestidas (M1), em relação aos valores determinados nas suspensões originais. Após a ressuspensão das micropartículas nanorrevestidas, o processo de desaglomeração leva à exposição de óxidos das superfícies do $\mathrm{SiO}_{2}$ diminuindo o potencial zeta.

A avaliação dos perfis de liberação in vitro da indometacina a partir das micropartículas nanorrevestidas (formulações M1 e M2), em meio intestinal simulado $(\mathrm{pH} \mathrm{6,8)}$ foi realizada em equipamento dotado de célula de fluxo, comparativamente, durante $200 \mathrm{~min}$. A partir deste tempo a concentração de fármaco nas alíquotas atingiu o limite de quantificação da indometacina pelo método analítico empregado. A modelagem matemática dos resultados (MicroMath Scientist $\left.{ }^{\circledR}\right)$ mostrou que a equação monoexponencial foi a que melhor descreveu o comportamento dos dados experimentais (Figura 4), com coeficiente de correlação $(r)$ próximo a 1 , semelhante ao que foi observado por Beck e colaboradores ${ }^{17}$ para a liberação de diclofenaco, em pH 7,4, a partir de micropartículas nanorrevestidas com nanocápsulas de Eudragit ${ }^{\circledR}$ S100. Através da modelagem foi possível calcular as constantes cinéticas da liberação do fármaco $(k)$. Para fins comparativos, foi também analisado o perfil de liberação da indometacina pura, que foi previamente cominuida. Os parâmetros calculados para todas as amostras estão apresentados na Tabela 5.

A indometacina pura apresentou uma velocidade de dissolução $\left(k=0,0222 \mathrm{~min}^{-1}\right)$ superior às velocidades de liberação da indometacina a partir das micropartículas ( $k=0,0135$ e $0,0106 \mathrm{~min}^{-1}$, para as formulações M1 e M2, respectivamente). Estes resultados demonstram a eficiência destes sistemas no controle da liberação da indometacina. Realizando-se a comparação entre as formulações preparadas a partir de nanocápsulas de diferentes polímeros, podese observar uma menor velocidade de liberação $\left(k=0,0106 \mathrm{~min}^{-1}\right)$ a partir das micropartículas nanorrevestidas com nanocápsulas de 


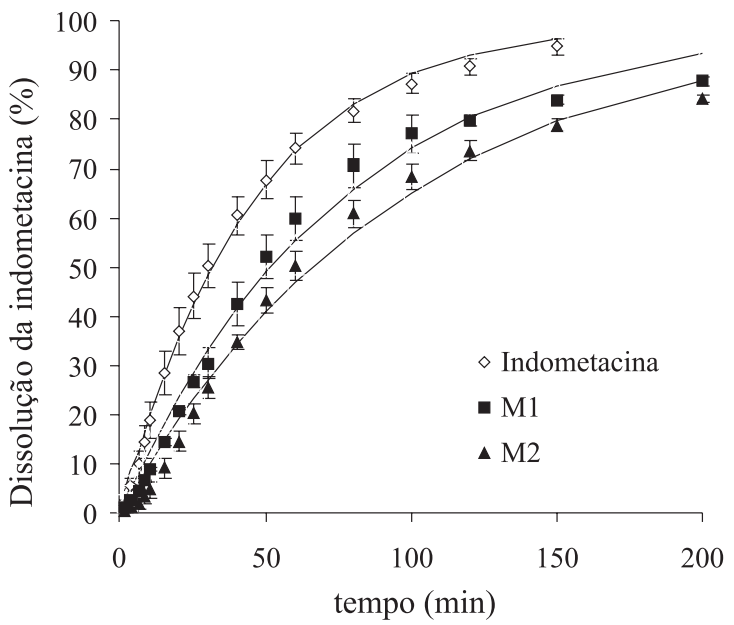

Figura 4. Perfis de dissolução e ajustes ao modelo monoexponencial da indometacina e das formulações M1 (PCL) e M2 (ERS). As linhas correspondem aos perfis modelados usando a equação monoexponencial

Tabela 5. Parâmetros do modelo monoexponencial para as formulações M1 (PCL), M2 (ERS) e indometacina triturada

\begin{tabular}{lc}
\hline Parâmetro & Média $\pm \mathrm{DP}$ \\
\hline Formulação M1 & \\
$\mathrm{k}\left(\mathrm{min}^{-1}\right)$ & $0,0135 \pm 0,0008$ \\
$\mathrm{r}$ & $0,9955 \pm 0,0018$ \\
$\mathrm{MSC}$ & $4,3630 \pm 0,3833$ \\
Formulação M2 & \\
$\mathrm{k}\left(\mathrm{min}^{-1}\right)$ & $0,0106 \pm 0,0006$ \\
$\mathrm{r}$ & $0,9959 \pm 0,0015$ \\
MSC & $4,1430 \pm 0,4119$ \\
Indometacina (matéria-prima) & - \\
$\mathrm{k}\left(\mathrm{min}^{-1}\right)$ & $0,0222 \pm 0,0028$ \\
$\mathrm{r}$ & $0,9985 \pm 0,0014$ \\
MSC & $5,5691 \pm 0,7073$ \\
\hline
\end{tabular}

Eudragit $^{\circledR}$ RS 100 (M2) em relação àquela obtida $\left(k=0,0135\right.$ min $\left.^{-1}\right)$ a partir da micropartículas nanorrevestidas com poli( $\varepsilon$-caprolactona) (M2). Essa redução nos valores da constante de liberação através do nanorrevestimento de micropartículas a partir de nanocápsulas também foi observada em estudo anterior, onde a liberação do diclofenaco a partir das micropartículas preparadas em duas etapas apresentava uma menor velocidade de liberação $\left(k=0,0283 \mathrm{~min}^{-1}\right)$ em relação a mistura física constituída do fármaco e do dióxido de silício coloidal $\left(k=0,0497 \mathrm{~min}^{-1}\right) \cdot{ }^{17}$ Adicionalmente, foram calculados os tempos de meia-vida (tempo necessário para que 50\% do fármaco fossem liberados ou dissolvidos). O tempo de meia-vida de dissolução para a indometacina pura foi de $31 \mathrm{~min}$, enquanto que as micropartículas nanorrevestidas apresentaram tempos de meia-vida de liberação do fármaco de 51 e 65 min, para as formulações M1 e M2, respectivamente. Pela comparação entre os tempos de meia-vida apresentados pelas diferentes formulações de micropartículas (M1 e M2) pode-se observar claramente o maior controle de liberação do fármaco a partir da formulação revestida com nanocápsulas de Eudragit ${ }^{\circledR} \mathrm{RS}$ 100 (M2). A possível interação molecular e consequiente formação de sal, entre o grupamento carboxílico da indometacina e o grupamento amônio quaternário do polímero Eudragit ${ }^{\circledR} \mathrm{RS}$ 100, também pode ser responsável pelo perfil de liberação mais lento da formulação M2. Esta hipótese de interação molecular entre a indometacina e o Eudragit ${ }^{\circledR}$ RS 100 é sustentada pelas alterações observadas no potencial zeta nas suspensões originais e após a ressuspensão das micropartículas em água, comentadas anteriormente.

O modelo de Korsmeyer-Peppas ${ }^{28,29}$ foi aplicado com o objetivo de descrever o mecanismo de liberação da indometacina a partir das micropartículas nanorrevestidas, através da análise dos valores de $n$. Foram encontrados valores de $n$ superiores a 0,85 (Tabela 6) para ambas as formulações, sugerindo uma liberação de acordo com um modelo não-Fickiano. Considerando a geometria esférica, esses valores indicam o modelo super caso-II para descrever o mecanismo de liberação da indometacina a partir das formulações M1 e M2. Neste caso, a velocidade de difusão do solvente para a partícula é muito maior que o relaxamento do polímero, sendo esse fator determinante da liberação. ${ }^{29} \mathrm{O}$ resultado é reforçado pelo baixo inchamento em meio aquoso apresentado pelos polímeros empregados neste estudo (dados não mostrados). Desta maneira, o modelo super caso-II pode ser descrito para as micropartículas nanorrevestidas preparadas em etapa única, as quais seriam constituídas de conjunto de partículas menores nanorrevestidas e aglomeradas, cujo mecanismo de liberação é a desaglomeração das micropartículas (erosão da partícula por fenômeno físico). Na Figura 5 está representada esquematicamente esta proposição de modelo de liberação da indometacina a partir das micropartículas nanorrevestidas preparadas em etapa única. A partir da entrada do meio de dissolução (penetração do solvente), as micropartículas sofrem desaglomeração, o que leva ao aumento de área superficial com conseqüente dissolução da indometacina. Como pode ser observado a partir dos resultados encontrados na avaliação dos perfis de liberação das formulações M1 e M2, o tipo de polímero utilizado na preparação das nanocápsulas poliméricas empregadas como material de revestimento influencia o acesso do solvente às micropartículas, alterando a velocidade de desaglomeração do sistema, e consequientemente, a velocidade de liberação do fármaco. Além disso, a interação da indometacina com os polímeros é de natureza físico-química diferente, podendo também influenciar na velocidade de liberação do fármaco.

Tabela 6. Parâmetros da Lei da Potência para as formulações M1 (PCL) e M2 (ERS)

\begin{tabular}{lc}
\hline Parâmetro & Média \pm DP \\
\hline Formulação M1 & \\
$\mathrm{a}$ & $0,9345 \pm 0,1049$ \\
$\mathrm{n}$ & $1,0245 \pm 0,0425$ \\
$\mathrm{r}$ & $0,9983 \pm 0,0003$ \\
MSC & $5,2142 \pm 0,1451$ \\
Formulação M2 & \\
$\mathrm{a}$ & $0,7122 \pm 0,2154$ \\
$\mathrm{n}$ & $1,0307 \pm 0,0733$ \\
$\mathrm{r}$ & $0,9934 \pm 0,0017$ \\
MSC & $3,8587 \pm 0,2408$ \\
\hline
\end{tabular}

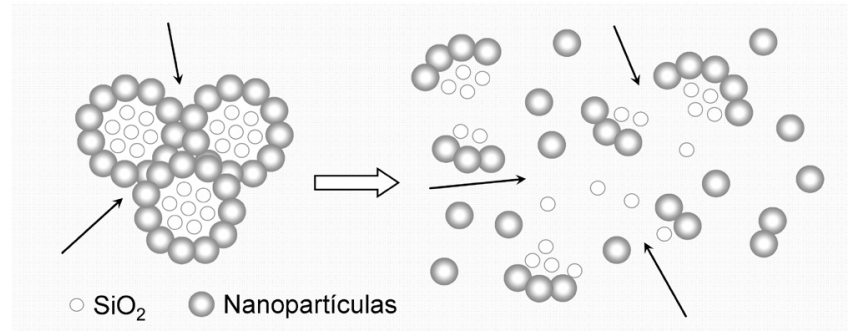

Figura 5. Esquema do mecanismo de desaglomeração a partir das micropartículas nanorrevestidas, as setas indicam a penetração do solvente 


\section{CONCLUSÃO}

Foi possível obter micropartículas nanorrevestidas preparadas em etapa única empregando um fármaco-modelo hidrofóbico, a indometacina, utilizando-se como polímeros a poli( $\varepsilon$-caprolactona) ou o Eudragit ${ }^{\circledast}$ RS 100. As micropartículas nanorrevestidas apresentaram aspecto pulverulento e através de MEV foram observadas partículas esféricas que apresentaram nanoestruturas adsorvidas a sua superfície, caracterizando o nanorrevestimento. Este recobrimento nanoestruturado levou a uma diminuição das áreas superficiais e dos volumes de poros destas micropartículas, quando comparadas ao suporte de secagem (Aerosil $\left.{ }^{\circledR} 200\right)$. Os estudos de liberação in vitro demonstraram a influência do polímero sobre as características de liberação da indometacina a partir das micropartículas nanorrevestidas, sendo que o modelo monoexponencial foi o mais adequado para descrever os perfis de liberação. O nanorrevestimento ocasionou um aumento no tempo de meia-vida de liberação da indomentacina quando comparada com a dissolução do fármaco puro. A formulação preparada a partir de nanocápsulas de Eudragit ${ }^{\circledR}$ RS 100 apresentou maior controle da liberação da indometacina, quando comparada àquela obtida a partir de micropartículas preparadas com a suspensão de poli(ع-caprolactona). A análise dos perfis através do modelo de Korsmeyer-Peppas mostrou que o mecanismo de liberação é não-Fickiano, sendo super caso-II. Um modelo de liberação da indometacina a partir das micropartículas nanorrevestidas baseado na desaglomeração das micropartículas foi proposto.

\section{AGRADECIMENTOS}

À CAPES, FAPERGS e Rede de Nanocosméticos/CNPq/MCT pelo financiamento e também ao Prof. Dr. E. V. Benvenutti pelo auxílio na determinação das áreas superficiais dos pós.

\section{REFERÊNCIAS}

1. Schaffazick, S. S.; Freitas, L. L.; Pohlmann, A. R.; Guterres, S. S.; Quim. Nova 2003, 26, 726.

2. Soppimath, K. S.; Aminabhavi, T. M; Kulkarni, A. R.; Rudziski, W. E.; J. Controlled Release 2001, 70, 1.

3. Müller, C. R.; Hass, S. E.; Bassani, V. L.; Guterres, S. S.; Fessi, H.; Peralba, M. C. R.; Pohlmann, A. R.; Quim. Nova 2004, 27, 555.

4. Guterres, S. S.; Fessi, H.; Barrat, G.; Puisieux, F.; Devissaguet, J. P.; Pharm. Res. 1995, $12,1$.

5. Guterres, S. S.; Müller, C. R.; Pohlmann, A. R.; Dalla Costa, T.; S.T.P. Pharma. Sci. 2001, 11, 229.

6. Guterres, S. S.; Weiss, V.; Lucca Freitas, L.; Pohlmann, A. R.; Drug Delivery 2000, 7, 195.
7. Müller, C. R.; Bassani, V. L.; Pohlmann, A. R.; Michalowski, C. B.; Petrovick, P. R.; Guterres, S. S.; Drug Dev. Ind. Pharm. 2000, 26, 343.

8. Pohlmann, A. R.; Weiss, V.; Mertins, O.; Pesce Da Silveira, N.; Guterres, S. S.; Eur. J. Pharm. Sci. 2002, 16, 305.

9. Müller, C. R.; Schaffazick, S. R.; Pohlmann, A. R.; De Lucca Freitas, L.; Pesce Da Silveira, N.; Dalla Costa, T.; Guterres, S. S.; Pharmazie 2001, 56, 864 .

10. Guterres, S. S.; Fessi, H.; Barrat, G.; Devissaguet, J. P.; Puisieux, F.; Int. J. Pharm. 1995, 113, 57.

11. Tewa-Tagne, P.; Briancon, S.; Fessi, H.; Int. J. Pharm. 2006, 325, 63.

12. Tewa-Tagne, P.; Degobert, G.; Briancon, S.; Bordes, C.; Gauvrit, J. Y.; Lanteri, P.; Fessi, H.; Pharm. Res. 2007, 24, 650.

13. Sham, J. O. H.; Zhang, Y.; Finlay, W. H.; Roa, W. H.; Löbenberg, R.; Int. J. Pharm. 2004, 269, 457.

14. Müller, C. R.; Tese de Doutorado, Universidade Federal do Rio Grande do Sul, Brasil, 2003.

15. Beck, R. C. R.; Pohlamnn, A. R.; Guterres, S. S.; J. Microencapsulation 2004, 21, 499.

16. Beck, R. C. R.; Pohlamnn, A. R.; Benvenutti, E. V.; Dalla Costa, T.; Guterres, S. S.; J. Braz. Chem. Soc. 2005, 16, 1233.

17. Beck, R. C. R.; Hass, S. E.; Guterres, S. S.; Re, M. I.; Benvenutti, E. V.; Pohlmann, A. R.; Quim. Nova 2006, 29, 990.

18. Beck, R. C. R.; Pohlmann, A. R.; Hoffmeister, C.; Gallas, M. R.; Collnot, E.; Schaefer, U. F.; Guterres, S. S.; Lehr, C. M.; Eur. J. Pharm. Sci. 2007, 67, 18 .

19. Fessi, H.; Puisieux, F.; Devissaguet, J. P.; Ammoury, N.; Benita, S.; Int. J. Pharm. 1989, 55, R1.

20. Farmacopéia Brasileira, IV ed., Atheneu: São Paulo, 1988.

21. Jambhekar, S.; Casella, R.; Maher, T.; Int. J. Pharm. 2004, 270, 149.

22. ICH-Harmonised Tripartity Guideline; Validation of Analytical Procedures: Methodology, IFPMA: Geneva 2005.

23. Brunauer, S.; Emmet, P. H.; Teller, E.; J. Am. Chem. Soc. 1938, 60, 309.

24. Barret, E. P.; Joyner, L. G.; Halenda, P. P.; J. Am. Chem. Soc. 1951, 73, 373.

25. Chen, J.; Davis, S. S.; J. Microencapsulation 2002, 19, 191.

26. United States Pharmacopeia, 29 ed., The United States Pharmacopeial Convention: Rockville, 2005.

27. Washington, C.; Int. J. Pharm. 1990, 58, 1.

28. Costa, P.; Lobo, J. M. S.; Eur. J. Pharm. Sci. 2001, 13,123.

29. Siepmann, J.; Peppas, N. A.; Adv. Drug Delivery Rev. 2001, 48, 139.

30. Schaffazick, S. R.; Pohlmann, A. R.; Mezzalira, G.; Gutterres, S. S.; J. Braz. Chem. Soc. 2006, 17, 562 .

31. Ubrich, N.; Schmidt, C.; Bodmeier, R.; Hoffman, M.; Maincent, P.; Int. J. Pharm. 2005, 288, 169.

32. Gibaud, S.; Al Awwadi, N. J.; Ducki, C.; Astier, A.; Int. J. Pharm. 2004, 269,491 